\title{
A New Method for Measuring Texture Regularity based on the Intensity of the Pixels in Grayscale Images
}

\author{
Khoerul Anwar \\ Departement of Computer \\ Science and Electronics \\ Universitas Gadjah Mada \\ Yogyakarta, Indonesia
}

\author{
Agus Harjoko \\ Departement of Computer \\ Science and Electronics \\ Universitas Gadjah Mada \\ Yogyakarta, Indonesia
}

\author{
Suharto Suharto \\ Departement of Computer \\ Science and Electronics \\ Universitas Gadjah Mada \\ Yogyakarta, Indonesia
}

\begin{abstract}
Texture regularity is one of the important visual characteristics. It can be used to determine differences in the surface between two objects. Several methods of measurement have been produced by previous researchers.However, in this paper the authors offer a new formula for calculating the regularity of texture features. Regularity is measured by the intensity of the pixels of grayscale images in a cross-diagonal position and the intensity of the pixels in an axis-ordinate position. The testing results of the new formula obtained good measurement accuracy. The linear test results using the human visual system worked. The observation of the human visual system suggested that a chessboard-image texture has a higher level of regularity than a bark-image texture. The results of measurement using the new formula showed that the value of the chessboard-texture regularity $(0.2490)$ was greater (a higher level of regularity) than that of the bark-texture regularity (0.0078).
\end{abstract}

\section{General term}

cross-diagonal, image, textures

Keywords

regularity; intensity of pixels; diagonal ordinate-axis (DOA)

\section{INTRODUCTION}

Knowing the specific characteristics of image textures is a fundamental problem in image processing, computer vision, object recognition and image reconstruction [1]. Each image has a characteristic loop, either regular or irregular one, called primitive texture [2][3] and the placement patterns in images containing one type of texture. Knowledge relating to the regularity of an object's texture is very useful. Exploration to acquire specific characteristics becomes important when there is a special image-processing task that requires specific features of an object. One of such characteristics is texture regularity.

Texture regularity is one of the important visual characteristics [4]. It can be used to determine differences in the surface between two objects. The measurement method is usually done by measuring the direct contact with the surface of the object or objects or by using the sensor surface. In the digital era, some measurement techniques have been developed with an object in the form of digital images. These techniques have several advantages, i.e. they are simple because they utilize mobile devices.

The visual texture in a digital image as an object of measurement is strongly influenced by the quality of the tools used during the acquisition of the object. One of the tools used to capture an image is a digital camera. The human visual system can work with and understand the texture of a digital image. In contrast to the working of the human visual, computing-based measuring tools (computers) have many limitations. One of the obstacles is when it is necessary to calculate the image texture with a random pattern. Another constraint is the texture of a digital image produced by the camera of a mobile device cannot show the structure of a specific texture. Especially for homogeneous image textures like meat, plain fabrics and carpets. Consequently, problems may arise when the computer system needs to identify several homogeneous images with similarities in texture.

Several previous researchers have undertaken a self-analysis of feature extraction. In some algorithms of computer vision and image processing, analyses simplify the assumptions made based on the regularity of the intensity of the local region pixel images [5]. However, the real object images often do not show the regularity and uniformity of the intensity of the region [6]. An image's surface has intensity variations that forms particular repeated patterns called visual texture [7]. Based on these intensity variations, the author assumes that texture regularity can be calculated using a specific formula. It is expected that the formula can be used as a distinguishing feature of an image.

This paper proposes a new method for calculating texture regularity based on the intensity of pixels in a diagonal axis matrix and the intensity of the pixels that are parallel to the axis of the coordinates $x$ and $y$. If the axes of the pixel intensities recur regularly, the visible image texture will form a certain pattern. The final calculation of the intensity of the pixels on the diagonal and cross- $\mathrm{x}$ and $\mathrm{y}$-coordinates then is set as the order value of a digital image.

The paper is organized as follows: Related works which explain the work of previous researchers discussing texture regularity presented in Section 2. The proposed method is presented in detail in Section 3. Then, result and discussion are presented in Section 4. The last part, i.e. the conclusion, is presented in Section 5.

\section{RELATED WORK}

Texture analyses, particularly to obtain texture features, have been undertaken by previous researchers. Analyses [8] generating texture features can be measured in terms of the uniformity, density, roughness, regularity, and intensity and focused on discrete tonal features and their spatial relationships. [9] In scientific work, the autocorrelation function of an image can be used to assess the regularity and smoothness/ roughness of an image's texture. In the autocorrelation model, the spatial organization is described by texture correlation coefficients to evaluate the spatial linear relationship between the primitive forms. The autocorrelation is calculated using a formula in Equation (1). 


$$
\rho(x, y)=\frac{\sum_{u=0}^{N} \sum_{v=0}^{N} I(u, v) I(u+x, v+y)}{\sum_{u=0}^{N} \sum_{v=0}^{N} I^{2}(u, v)}
$$

Chetverikov [10] used regularity to find damage to the texture. The approach is based on the basic assumption that defect-free patterns show some homogeneous periodicity. There are two important things to find damage to the texture, the regularity and to detect abnormalities in the texture (outlier detection). Damage is measured based on the periodization of the autocorrelation function of image $I(m, n)$ and the distance vector $(\mathrm{dx}, \mathrm{dy})$.

Leu [11] performed calculations based on the correlation between the texture profile and the texture regularity. The correlation between the height of the peak point $(\mathrm{C} 1)$ and the next highest value (C2) in a gradient of autocorrelation. The value of the order is defined as $\mathrm{C} 2 / \mathrm{C} 1$, for both of them are positive. The upper limit is the degree of order by 1 . If the regularity is negative, then the degree of regularity is 0 . He defines the degree of order as

$$
\text { regularity }=\left\{\begin{array}{l}
\frac{C_{2}}{C_{1}} \text { when } C_{2} \geq 0 \\
0 \text { when } C_{2}<0
\end{array}\right.
$$

Ye [12] analyzes texture regularity based on local pixel intensity (window intensity regularity). The basic idea that when the intensity distribution on the two sides of similar pixels, pixels are located in an area considered an irregular texture and there is no change in edge pixels. Texture regularity is used to distinguish the texture of the original object from the texture of the object after the deformed shape [13].

\section{PROPOSED METHODE}

The new method of measuring the regularity offered has a different regularity measurement concept from that offered by previous researchers. The author offers a new way of calculating the order based on the intensity of the pixels of an image matrix, especially on the main diagonal parallel pixel matrix and the $\mathrm{x}$ and $\mathrm{y}$-coordinates. The detailed explanation of the method is presented in Subsection 3.3, New Method. The method has offered a three-stage process. The first process is to convert RGB images to grayscale ones. Then, the second process is to filter the image noise and the third stage is to calculate the regularity of the proposed method.

\subsection{Image Conversion}

Conversion from an RGB image to a grayscale image to support the image analysis process at the level of grayscale images is shown in Figure 1.

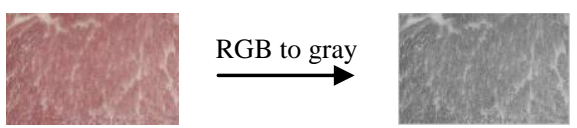

Figure 1. RGB to grascale:

Figure 1 shows changes in the color of an image into a grayscale image. The image to the left is the original image while the right image is the conversion result.

\subsection{Filter}

An analyzed image refers to a digital image of beef and pork captured using a mobile-device camera. The visual texture of beef and pork digital images does not have any specific texture pattern. Therefore, to obtain maximum information about the texture, the visual texture of the image information needs to be optimized. Gabor filter-based feature extractors can be interpreted as a nonlinear function that maps an image/ images of the original space to the feature space, here each image is represented by their own features [14]. Gabor filters have the ability necessary for such optimization. Gabor filters provide intensity extraction of multiple orientations. Consider obtaining optimal texture information on the paper used and select the Gabor filter orientation by $0^{\circ}$. The underlying reason for the selection was based on test results for the Gabor filter orientation by $0^{\circ}, 45^{\circ}, 90^{\circ}$ and $135^{\circ}$ which showed that the $0^{\circ}$ orientation generated optimal results compared with the others, as shown in Figure 2..

\begin{tabular}{|c|c|c|c|c|}
\hline Original & $0^{\circ}$ & $45^{\circ}$ & $90^{\circ}$ & $135^{\circ}$ \\
\hline & & & & \\
& & & & \\
\hline
\end{tabular}

Figure 2. Gabor filter

Figure 2 shows the results of Gabor filters with the best output quality of the process in $0^{\circ}$ orientation.

\subsection{Diagonal-Ordinat Axis (DOA)}

results. The distribution of the intensity of the image can be used to obtain texture features. The type of popular features used was the GLCM [8]. This paper offers regularity as a new feature (novelty) using the formulas in Equations (1), (2) and (3). This feature was calculated based on the shared values of pixels in the same position of the two vectors. While the vector obtained from the values of pixels in a matrix on the diagonal axis and the axis-cmatrix. The composition of the matrix pixel values in the image matrix is shown in Figure 3. The matrix had a dimension of $5 \times 5$ (hxv). In this research, the matrix was set so that the number of rows and columns is in an odd number.

$$
M_{h \times v}=\left[\begin{array}{ccccc}
m_{11} & m_{12} & m_{13} & \ldots & m_{1 v} \\
m_{21} & m_{22} & m_{23} & \ldots & m_{2 v} \\
m_{31} & m_{32} & m_{33} & \ldots & m_{3 v} \\
\ldots & \ldots & \ldots & \ldots & \ldots \\
m_{h 1} & m_{h 2} & m_{h 3} & \cdots & m_{h v}
\end{array}\right]
$$

Figure 3. Matrix image

The values of each vector element obtained from reading the pixel values in the cross-diagonal and axis-ordinate positions with a darker color are shown in Figure 4.
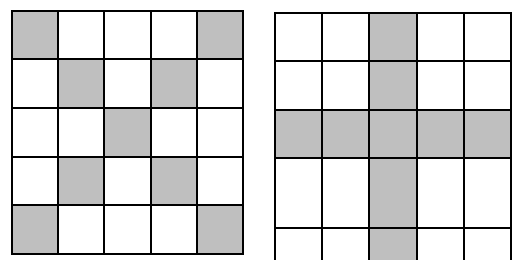

Figure 4. The position of the distribution of pixels. a) cross diagonal, b) ordinate axis

The calculation of the proposed regularity refers to the act of measuring the regularity of the pixels in the cross-diagonal position and the regularity of the pixels in the axis-ordinate position. The position is shown in Figure 4. The value iteration of a particular pixel in the cross-diagonal position or in the axis-ordinate position will form a particular object. The 
recurrence illustration of such pixels as shown in Figure 5 shows the distribution of the pixel values of grayscale images by looping the rows and columns of the images on a regular basis. Like the arrangement of pixels which displays regularity. Thus, the pixel array can be used as a special feature. In this section, the analysis of pixel distribution in an image was undertaken in order to determine the texture regularity size based on the distribution of pixels. To calculate the regularity of the pixel distribution, this analysis was carried out in two stages, namely recording pixels and calculating the regularity.

\begin{tabular}{|l|l|l|l|l|}
\hline 13 & 10 & 11 & 10 & 13 \\
\hline 10 & 22 & 20 & 22 & 10 \\
\hline 11 & 20 & 10 & 20 & 11 \\
\hline 10 & 22 & 20 & 22 & 10 \\
\hline 13 & 10 & 11 & 10 & 13 \\
\hline
\end{tabular}

\section{Figure 5. The pixel values of DOA}

In this section, the analysis of pixel distribution in an image was undertaken in order to determine the texture regularity size based on the distribution of pixels. To calculate the regularity of the pixel distribution, this analysis was carried out in two stages, namely recording pixels and calculating the regularity. The stage of recording pixel distribution consisted of two processes. The first one was the process of recording pixels in the diagonal (Figure 4.a) major matrix subsequently referred to as the vector $r_{1}$. Referring to Figure 3, the members of vector $r_{1}$ are $\left\{m_{11}, m_{22}, m_{33}, \ldots, m_{h v}\right\}$ while the second diagonal side is referred to as the vector $\boldsymbol{r}_{2}$ with members of vector $\boldsymbol{r}_{2}$, namely $\left\{\mathrm{m}_{\mathrm{h} 1}, \ldots, \mathrm{m}_{33}, \ldots, \mathrm{m}_{1 \mathrm{v}}\right\}$. The second process was recording pixels in the middle column of the matrix (parallel to the $\mathrm{x}$-axis) and in the middle row of the matrix (parallel to the $y$-axis). Pixel distribution in the middle column of the matrix is referred to as vector $r_{3}$. Referring to Figure 3, the members of vector $r_{3}$ are $\left\{m_{13}, m_{23}, m_{33}, \ldots, m_{h 3}\right\}$ and the pixel distribution in the middle row of the matrix is referred to as vector $r_{4}$, where the members of vector $r_{4}$ are $\left\{m_{31}, m_{32}\right.$, $\left.m_{33}, \ldots, m_{3 v}\right\}$.

The stages of regularity calculation began with calculating regularity of the diagonal vectors $\mathbf{r}_{1}$ and $\mathbf{r}_{2}$ and matching the elements of vectors $\boldsymbol{r}_{\boldsymbol{l}}$ and $\boldsymbol{r}_{2}$. The result of this process is referred to as the diagonal matrix of the order value and symbolized by $\boldsymbol{R}_{\boldsymbol{I}}$. Then, the regularity of the middle column and the middle row (Figure $4 \mathrm{~b}$ ) of the matrix, namely $\boldsymbol{r}_{3}$ and $\boldsymbol{r}_{4}$, was calculated by matching the elements of vectors $\boldsymbol{r}_{3}$ and $\boldsymbol{r}_{4}$. The result obtained from this process is referred to as the order value of the axis-ordinate position and symbolized by $\boldsymbol{R}_{2} . \boldsymbol{R}_{\boldsymbol{1}}$ was calculated using Equation (1) while $\boldsymbol{R}_{\mathbf{2}}$ was used to calculate Equation (2). If the elements of vectors $r 1$ and $r 2$ have the same values in the index of vector $i$, and the elements of vectors $r 3$ and $r 4$ have the same values in the index of vector $\mathrm{j}$, it means that the matrix has a regular element.

The logical operators to match the regularity of pixels in $R_{I}$ and $R_{2}$ were NOT and XOR (XNOR). The XOR logical operator will be true or equal to 1 , if the values being compared are different and will be worth one or 0 , if the values being compared are equal. While based on the same XOR operation, the value is 0 , then the necessary negation of XOR or XNOR known changes 0 to 1 . Therefore, for the same pixels, they will be equal to 1 . The technique to calculate the pixel value of the order was based on the value of the same pixel in the same position of two vectors. For the operation of $a X N O R b$ with two variables of the same value, the result is 1 . While the operation of $a$ XNOR $b$ with different variable values, the result is 0 . This is shown in Figure 6.

\begin{tabular}{|c|c|c|c|}
\hline No & $\mathrm{a}$ & $\mathrm{b}$ & XNOR \\
\hline 1 & 11 & 11 & 1 \\
\hline 2 & 13 & 10 & 0 \\
\hline 3 & 10 & 13 & 0 \\
\hline 4 & 0 & 0 & 1 \\
\hline
\end{tabular}

Figure 6. XNOR logic

$\boldsymbol{r}_{1}, \boldsymbol{r}_{2}, \boldsymbol{r}_{3}, \boldsymbol{r}_{4}$ are a vector. Based on these vectors, the level of order cross-pixels of the diagonal $\left(\boldsymbol{r}_{1} \boldsymbol{r}_{2}\right)$ is the accumulated value of the operating $\boldsymbol{r}_{1} X N O R \boldsymbol{r}_{2}$, so it can mathematically be formulated as Equation (1),

$$
R_{1}=\sum_{\mathrm{i}=}^{\mathrm{n}} \neg\left(\mathrm{r}_{1 \mathrm{i}} \mathrm{XOR}_{2 \mathrm{i}}\right)
$$

As for the regularity of pixels in the vertical-horizontal position $\left(\boldsymbol{r}_{3} \boldsymbol{r}_{4}\right)$, the accumulated value of the operating $\boldsymbol{r}_{3}$ $\mathrm{XNOR} \mathbf{r}_{4}$ can mathematically be formulated as Equation (2),

$$
R_{2}=\sum_{\mathrm{i}=}^{\mathrm{n}} \neg\left(\mathrm{r}_{3 \mathrm{i}} \mathrm{XOR}_{4 \mathrm{i}}\right)
$$

Finally, an image's regularity is obtained by calculating the average of $R_{1}$ and $R_{2}$. The formulation is presented below

$$
R_{g}=\frac{R_{1}+R_{2}}{2 * N}
$$

In Equation (3), $R_{g}$ is used to calculate the texture regularity of the $h x v$ image size. $R_{l}$ refers to the regularity of the value of vectors $r_{1}$ and $r_{2}, R_{2}$ refers to the regularity of vectors $r_{3}$ and $r_{4}$. The symbol $N$ indicates the length of the vector.

\subsection{Algorithm}

The calculation to generate the regularity of grayscale images based on Equation (3) can be done using the steps or algorithm shown in Figure 7.

1. Read the grayscale image intensity values

2. Reading the image matrix size ( $h x v)$

3. Determine the middle row and the middle column of the matrix

4. Determine the vector $r_{1}, r_{2}, r_{3}$ and $r_{4}$

for $i=1:$ matrixlength

$$
\begin{aligned}
& \text { vektor } r_{1}(i)=I(i, i) ; \\
& \text { vektor } r_{2}(i)=I(c-i, i) ; \\
& \text { vektor } r_{3}(i)=I(h, i) ; \\
& \text { vektor } r_{4}(i)=I(i, v) ;
\end{aligned}
$$

end

5. Calculate the regularity of each vector using equation (1) and (2)

6. Calculate the regularity of the image using the equation (3)

Figure 7. Regularity Algorithm 


\section{RESULT AND DISCUSSION}

The new method was tested using beef and pork images captured using a 5 megapixels mobile-device camera. Then, it was tested using some digital images freely downloaded. The beef and pork images have a similar texture (homogeneous) while the texture of freely-downloaded images does not (heterogeneous). The first test was performed on two images with a homogeneous texture and the second one was performed on the images with a heterogeneous texture. Moreover, the sensitivity to the light intensity was also examined..
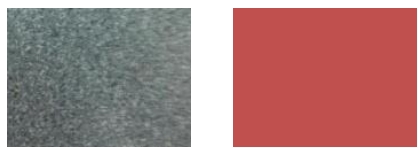

Ceramic Floor

Red color

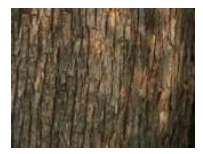

Pork

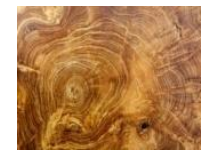

teak trunk

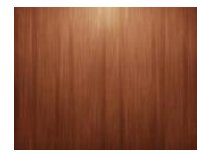

plank

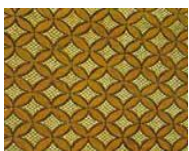

batik

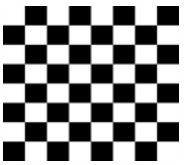

Chessboard

Figure 8. Texture configuration

\subsection{Pork and Beef Regularity}

The images captured using a 5 megapixels mobile-device camera were tested. Results of the test are as shown in Table 1.

Table 1. The regularity value of beef and pork images

\begin{tabular}{|l|l|l|}
\hline No & Image name & Regularity \\
\hline 1 & Beef & 0.0118 \\
\hline 2 & Pork & 0.0314 \\
\hline
\end{tabular}

Table 1 presents the regularity values of the beef and pork images by 0.0118 and 0.0314 , respectively. These results indicate that the regularity of the beef image is smaller than that of the pork image or, on the other words, the texture regularity of the pork image is higher than that of the beef image..

\subsection{Another Images}

The proposed method was also tested using several images with a different texture. The image types and the test results are presented in Table 2.

Table 2. Regularity some kind of texture image

\begin{tabular}{|l|l|l|}
\hline No & Image name & Regularity \\
\hline 1 & The texture of the bark & 0.0078 \\
\hline 2 & Texture of teak trunk & 0.0150 \\
\hline 3 & Texture chessboard & 0.2490 \\
\hline 4 & Texture ceramic Floor & 0.0353 \\
\hline 5 & Texture red & 1 \\
\hline 6 & Texture batik & 0.0118 \\
\hline 7 & Texture wooden plank & 0.0196 \\
\hline
\end{tabular}

Table 2 shows that several visual images have an irregular texture, namely images number 3,4 and 5 with a regularity value by $0.0353,0.2490$ and 1 , respectively. In addition, some visual images tend to be less irregular in their texture, namely images number 1 and 2 with a regularity value by 0.0150 and
0.0078 , respectively. Visual images with a higher degree of texture regularity generate a greater regularity value than that of the visual images with an irregular texture.

\subsection{Light Intensity}

The test on the effect of light intensity on the surface of an image was conducted in order to determine the regularity of an image. The test data consisted of the chessboard image, Lux meter (i.e., an intensity measuring instrument), and a 5MP mobile-device camera. There were a total of 10 variations of intensity in order of magnitude, namely: $17,31,39,43,49,51$, $58,76,105$, and 113 . The distance was $6 \mathrm{~cm}$ away from camera with the image dimensions of $2592 \times 1944$. The regularity values of ten images with a different level of light intensity were calculated. The results, in order of the light intensity magnitude, were $0.0450,0.0235,0.0216,0.0137,0.0235$, $0.0294,0.0235,0.0412,0.0196,0.0451$ and 0.0176 . Figure 8 presents a graph illustrating the relationship between the light intensity and the regularity value..

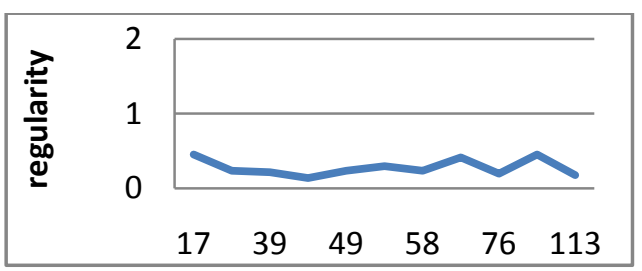

Figure 8. lighting intensity

Figure 8 shows a graph describing the effect of the light on the regularity of the chessboard in different light intensities. The yaxis indicates regularity values while the $\mathrm{x}$-axis indicates light intensity values. Figure 8 shows different light intensity values and the regularity values are also different, meaning that regularity is influenced by the intensity of light on the surface of an image. 


\section{CONCLUSION}

Based on the three types of tests described previously, the following conclusions can be drawn: the new method proposed for measuring regularity managed to show the difference in regularity between digital images and images with a similar texture, as shown in Table 1. The new method also managed to distinguish the regularity values of visual images with a heterogeneous texture (Table 2). Images number 4 and 5 show a larger value than that of the visual images with a less regular texture, i.e. number 1 and 2 , as shown in Table 2. However, the regularity value is also influenced by the light intensity on the surface of an image. Based on the three tests and the obtained results, the new method can be used as a new feature for the image-texture analysis.

Further research can develop feature extraction methods to obtain the texture using the features of regularity

\section{ACKNOWLEDGMENT}

The author would like to thank the Ministry of Research and Higher Education of the Republic of Indonesia for financial support provided for this research dissertation.

\section{REFERENCES}

[1] M. Lukashevich, and R. Sadykhov, 2012, Texture Analysis: Algorithm for Texture Teatures Computation. IV International Conference "Problems of Cybernetics and Informatics" (PCI'2012), IEEE. Baku, Azerbaijan , 161163.

[2] S. Varadarajan, and L. J. Karam, 2014, Adaptive Texture Synthesis Based On Perceived Texture Regularity. Sixth International Workshop on Quality of Multimedia Experience (QoMEX), IEEE,2014, 76-80.

[3] C. Zheng, D.W. Sun, and L. Zheng, 2007, A new region-primitive method for classification of colour meat image texture based on size, orientation, and contrast, Meat Science, 620-627.

[4] O. Gyuhwan, S. Lee, and S.Y. Shin, 1999, Fast determination of textural periodicity using distance matching function, Pattern Recognition Letters, 191-197.
[5] R.F. Wang, W.Z. Chen, S.Y. Zhang, Y. Zhang, and X.Z. Ye, 2008 ,Similarity-based denoising of point-sampled surfaces, Journal of Zhejiang University Science A , 807815 .

[6] M. Deswali, and N. Sharma, 2014, A Simplified Review on Fast HSV Image Color and Texture Detection and Image Conversion Algorithm, International Journal of Computer Science and Mobile Computing, 1216-1222.

[7] A.H. Rasal, and J. Rangole, 2015, A Review On Approaches To Texture Analysis Of Seabed Images. International Journal of Informative \& Futuristic Research, 2347-1697.

[8] R. M Haralick, 1979, Statistical and structural approaches to texture. Proceedings of the IEEE 67, IEEE, 786-804.

[9] G.N. Srinivasan, and G. Shobha, 2008, Statistical Texture Analysis. Proceedings Of World Academy Of Science, Engineering And Technology Volume 36 WASET, 12641269.

[10] D. Chetverikov, and A. Hanbury, 2002, Finding defects in texture using regularity and local orientation, Pattern Recognition, pp $2165-2180$

[11] J.G. Leu, 2001, On Indexing The Periodicity of Image Texture. Image and Vision Computing, 987-1000.

[12] Q. Ye, W. Gao, and W. Wang, 2003, A New TextureInsensitive Edge Detection Method, CICS-PCM, ingapure, IEEE, 768-772.

[13] D. Liu, Y. Xu, U. Quan, and P.L Callet, 2014, Reduced reference image quality assessment using regularity of phase congruency. Signal Processing, Image Communication, 844-855.

[14] W. Li, K. Mao, H. Zhang, and T. Chai, 2010, Selection of Gabor Filters for Improved Texture Feature Ekstraction. Proceedings of 2010 IEEE 17th International Conference on Image Processing, Hongkong: IEEE, 361-364. 